\title{
Diferentes Balanços Catiônicos-Aniônicos da Dieta de Vacas da Raça Holandesa ${ }^{1}$ \\ Laudí Cunha Leite ${ }^{2}$, José Luciano Andriguetto ${ }^{3}$, Meiby Carneiro de Paula ${ }^{4}$, Rita Maria Venancio Mangrich Rocha ${ }^{5}$
}

\begin{abstract}
RESUMO - Os objetivos deste trabalho foram avaliar os efeitos de três dietas, com balanços catiônicos-aniônicos da dieta (BCAD) $\mathrm{de}+122,1,-8,8 \mathrm{e}-110,4 \mathrm{mEq} / \mathrm{kg}$ de MS, fornecidas durante quatro semanas antes do parto para os grupos 1,2 e 3 , respectivamente, sobre as concentrações de cálcio iônico $(\mathrm{CaI})$ e cálcio total $(\mathrm{CaT})$ plasmáticos, a incidência de hipocalcemia subclínica e o pH urinário. Foram utilizadas 21 vacas em um delineamento experimental inteiramente casualizado no esquema de parcela subdividida, com sete repetições. As amostras de plasma foram colhidas no início da dieta, até 12 horas após o parto e com 1, 2, 3 e 7 dias depois do parto, denominadas de períodos de colheitas 1, 2, 3, 4, 5 e 6, respectivamente. As amostras de urina foram colhidas semanalmente e agrupadas em três períodos para a análise, sendo o período 1 correspondente ao início do experimento e os períodos 2 e 3 as colheitas realizadas entre 20 e 11 dias e entre 10 e 1 dias antes do parto, respectivamente. Não houve diferença na incidência de hipocalcemia subclínica entre os grupos. Os níveis de $\mathrm{CaI}$ e CaT plasmáticos diferiram significativamente entre os períodos de colheitas. O dia do parto teve os menores valores de $\mathrm{CaT}(7,52 \mathrm{mg} / \mathrm{dL})$ e de $\mathrm{CaI}(3,974 \mathrm{mg} / \mathrm{dL})$. O pH urinário diferiu significativamente entre as dietas, períodos de colheitas e interação dieta e períodos de colheitas. O grupo 3 apresentou o menor valor de pH urinário no período de colheita 2 (7,238) em relação ao período 1 $(8,094)$ e em relação ao grupo $1(8,045)$ para o mesmo período. O fornecimento da dieta aniônica não teve efeito sobre as concentrações de $\mathrm{CaI}$ e CaT, reduziu o pH urinário e não impediu a ocorrência de hipocalcemia subclínica.
\end{abstract}

Palavras-chave: dieta aniônica, paresia da parturiente, período de transição, vacas secas

\section{Decreasing Dietary Cation-Anion Balances in Holstein Cows}

\begin{abstract}
The objective of the present experiment was to evaluate the effect of three different dietary levels of cation-anion balance (DCAB) $(+122.1 ;-8.8$; and $-110.4 \mathrm{mEq} / \mathrm{kg} \mathrm{DM})$ offered for four weeks to groups 1,2 and 3 , respectively, on the plasma levels of ionized calcium ( $\mathrm{iCa}$ ) and total calcium $(\mathrm{tCa})$, the incidence of subclinic hypocalcemia and the urine $\mathrm{pH}$. Twenty-one Holstein cows were assigned to a split plot in time design with seven replications; DCAB was effects in the main plot, and sampling period was effects in the subplot. Plasma samples were collected from the jugular vein at the beginning of treatment and until 12 hours, 1, 2, 3 and 7 days after calf birth (sampling periods 1, 2, 3, 4, 5 and 6, respectively). Urine samples were taken weekly from the beginning of treatment until delivery and were grouped, for the analysis, in three periods. Period 1 is the initiation of the treatment, period 2 extends from 11 to 20 days prepartum, and period 3 extends from 10 days before until the day of delivery. Incidence of subclinic hypocalcemia did not differ between groups. There was no significant effect of treatment or of the interaction between treatment and sampling period on the plasma levels of iCa or $\mathrm{tCa}$, but there was a significant difference on those levels for the sampling period. The lowest levels of both iCa $(3.974 \mathrm{mg} / \mathrm{dL})$ and $\mathrm{tCa}(7.52 \mathrm{mg} / \mathrm{dL})$ were observed at the day of delivery. Urine $\mathrm{pH}$ varied significantly among treatments, sampling period and their interaction. Group 3 showed the lowest $\mathrm{pH}$ value at sampling period 2 (7.238) compared to period 1 (8.094) and to group 1 (8.045) for the same period. Feeding an anionic diet, by the inclusion of anionic salts to the concentrate feed, had no effect on plasma concentrations of $\mathrm{iCa}$ and $\mathrm{tCa}$, reduced urine $\mathrm{pH}$ and did not prevent the occurrence of subclinic hypocalcemia.
\end{abstract}

Key Words: anionic diet, dry cow, milk fever, transition period

\section{Introdução}

O início da lactação impõe à vaca grande demanda por cálcio $(\mathrm{Ca})$. Algumas vacas não conseguem se adaptar a esta súbita demanda e entram em um quadro de hipocalcemia, chamado de paresia da parturiente (Goff, 1992).
A paresia da parturiente reduz em $14 \%$ a produção total de leite na lactação em que ela ocorre (Block, 1984), tem uma associação com outras doenças do período periparto, como distocia, retenção de placenta, cetose e mastite (Curtis et al., 1983), reduz a vida produtiva da vaca leiteira em 3,4 anos (Payne, 1968, citado por Curtis et al., 1983) e apresenta uma

\footnotetext{
${ }^{1}$ Parte da dissertação de mestrado do primeiro autor, Pós-Graduação em Ciências Veterinárias da UFPR

${ }^{2}$ Méd. Vet., M.Sc., Rua Aristides França, 202, CEP: 83035-170 - São José dos Pinhais-PR. E.mail: laudileite@zipmail.com.br

${ }_{3}^{3}$ Méd. Vet., Ph.D., Professor Departamento de Zootecnia da UFPR. E.mail: jluciano@agrarias.ufpr.br

${ }^{4}$ Zootecnista, M.Sc., Professora Faculdades Integradas "Espírita".

5 Méd. Vet., M.Sc., Professora PUC-PR.
} 
incidência de 8 a $9 \%$ em rebanhos leiteiros dos Estados Unidos (Sanchez et al., 1992, citados por Sanchez \& Blauwiekel, 1999). No Brasil, Ortolani (1995), trabalhando com um único rebanho, revelou incidência de $4,25 \%$.

A paresia da parturiente apresenta uma forma subclínica, em que as vacas apresentam hipocalcemia (concentração de cálcio iônico $<4,0 \mathrm{mg} / \mathrm{dL}$ ) à parição, mas não demonstram sinais clínicos. A hipocalcemia subclínica apresenta maior incidência (Beede et al., 1992, citados por Sanchez \& Blauwiekel, 1999) e, devido à falta de diagnóstico e conseqüente ausência no tratamento, pode ocasionar maiores prejuízos à bovinocultura leiteira.

$\mathrm{O}$ uso de sais aniônicos ou a manipulação do balanço catiônico-aniônico da dieta (BCAD) tem sido utilizado como um método efetivo na manutenção dos níveis de Ca durante a parição, prevenindo a paresia da parturiente (Block, 1984).

O BCAD é determinado pelo balanço entre os cátions fixos totais (biodisponíveis e não metabolizáveis) e os ânions fixos totais presentes na dieta (Wang et al., 1996). Este conceito é baseado na teoria de íons fortes (Stewart, 1983), na qual qualquer entrada de íons no corpo leva a alteração do equilíbrio ácido-básico.

Os minerais sódio $(\mathrm{Na})$, potássio $(\mathrm{K})$, cloro $(\mathrm{Cl}) \mathrm{e}$ enxofre (S) têm sido utilizados no cálculo do BCAD porque sua importância no metabolismo está associada à sua participação no balanço osmótico, balanço ácido-básico, mecanismo de bombeamento e integridade de membranas (Block, 1994). O papel dos outros minerais, como $\mathrm{Ca}$, fósforo $(\mathrm{P})$ e magnésio $(\mathrm{Mg})$, no equilíbrio ácido-básico ainda não está completamente entendido. Goff (1992) recomenda a utilização destes minerais no cálculo do $\mathrm{BCAD}$, mas as fórmulas são mais complexas e não têm sido utilizadas na grande maioria dos trabalhos.

A dieta aniônica provoca acidose metabólica moderada, que, por sua vez, aumenta a capacidade de resposta dos tecidos ao hormônio PTH (Beck \& Webster, 1976; Goff et al., 1991) e aumenta o fluxo de Ca através do pool de Ca trocável (Takagi \& Block, 1991), habilitando a vaca a manter os níveis de Ca normal durante a parição.

O valor do BCAD deve ficar entre -100 e $-150 \mathrm{mEq} / \mathrm{kg}$ de matéria seca (MS) para a manutenção da concentração plasmática de Ca dentro dos limites normais (Davidson et al., 1995; Moore et al., 2000).

\footnotetext{
R. Bras. Zootec., v.32, n.5, p.1259-1265, 2003
}

Um método fácil e barato de monitorar o efeito dos sais aniônicos sobre o equilíbrio ácido-básico é a medição do potencial hidrogeniônico $(\mathrm{pH})$ urinário. Segundo Davidson et al. (1995), o pH urinário deve ficar entre 5,5 e 6,5 para que a dieta aniônica tenha o efeito desejado. Já Jardon (1995) recomenda valores de $\mathrm{pH}$ urinário entre 6 e 7.

Os objetivos do presente trabalho foram avaliar os efeitos de diferentes balanços catiônicos-aniônicos da dieta sobre os níveis de cálcio iônico (CaI) e cálcio total (CaT) plasmáticos, a incidência de hipocalcemia subclínica e o pH urinário em vacas da raça Holandesa.

\section{Material e Métodos}

O experimento foi conduzido na Chácara Vitória, localizada no município de Palmeira, Paraná, no período de julho a novembro de 2001.

Foram utilizadas 21 vacas da raça Holandesa com duas ou mais lactações, em um delineamento experimental inteiramente casualizado, com três tratamentos e sete repetições no esquema de parcela subdividida, onde as dietas foram estudadas nas parcelas e os períodos de colheitas nas subparcelas.

A hipocalcemia subclínica foi determinada pela concentração de CaT menor do que 7,5 mg/dL (Goff \& Horst, 1998) ou concentração de CaI menor do que 4,0 mg/dL (Beede et al., 1992, citados por Sanchez \& Blauwiekel, 1999).

A dieta dos animais teve início quatro semanas antes da data prevista para o parto e foi composta por uma mistura de silagem de milho e de silagem préseca de azevém preparada em vagão misturador e fornecida uma vez ao dia, e de um concentrado a base de milho e soja, com ou sem adição de sais aniônicos, fornecido duas vezes ao dia. A alimentação foi fornecida para atender ou exceder os requerimentos para vacas secas do NRC (2001). As dietas pré-parto foram isoprotéicas $(16,1 \%$ de proteína bruta - PB) e isocalóricas (1,82 Mcal ELl). Após o parto, as vacas seguiam para o lote de vacas recém-paridas, onde recebiam uma dieta catiônica igual para todos os grupos. Pode-se observar a composição mineral das dietas pré-parto na Tabela 1.

Os tratamentos diferiram entre si pela concentração de sais aniônicos no concentrado, sendo o grupo 1 sem a adição de sais, o grupo 2 com a adição de $50 \mathrm{~g}$ de $\mathrm{CaCl}_{2} * 2 \mathrm{H}_{2} \mathrm{O}$ e $110 \mathrm{~g}$ de $\mathrm{MgSO}_{4} * 7 \mathrm{H}_{2} \mathrm{O}$ e o grupo 3 com $130 \mathrm{~g} \mathrm{de} \mathrm{CaCl}_{2} * 2 \mathrm{H}_{2} \mathrm{O}$ e $130 \mathrm{~g}$ de $\mathrm{MgSO}_{4} * 7 \mathrm{H}_{2} \mathrm{O}$. 
Tabela 1 - Composição mineral das dietas Table 1 - Mineral composition of the diets

\begin{tabular}{lccc}
\hline Minerais (\%) & $\begin{array}{c}\text { Grupo 1 } \\
\text { Minerals (\%) }\end{array}$ & $\begin{array}{r}\text { Grupo 2 } \\
\text { Group 2 }\end{array}$ & $\begin{array}{r}\text { Grupo 3 } \\
\text { Group 3 }\end{array}$ \\
\hline $\mathrm{Ca}$ & 0,67 & 0,68 & 0,70 \\
$\mathrm{P}$ & 0,33 & 0,33 & 0,33 \\
$\mathrm{Mg}$ & 0,32 & 0,31 & 0,32 \\
$\mathrm{Na}$ & 0,12 & 0,12 & 0,12 \\
$\mathrm{~K}$ & 1,24 & 1,23 & 1,22 \\
$\mathrm{C}$ & 0,33 & 0,52 & 0,83 \\
$\mathrm{~S}$ & 0,25 & 0,37 & 0,39 \\
$\mathrm{BCAD}^{1}$ & 122,1 & $-8,82$ & $-110,41$ \\
$D C A B^{1}$ & & &
\end{tabular}

${ }^{1} \mathrm{mEq}(\mathrm{Na}+\mathrm{K})-(\mathrm{Cl}+\mathrm{S}) / \mathrm{kg}$ de $\mathrm{MS}$.

${ }^{1} \mathrm{mEq}(\mathrm{Na}+\mathrm{K})-(\mathrm{Cl}+\mathrm{S}) / \mathrm{kg} D \mathrm{DM}$

O BCAD foi de $+122,1,-8,82$ e $-110,41 \mathrm{mEq} / \mathrm{kg}$ de MS, para os grupos 1, 2 e 3, respectivamente.

$\mathrm{O}$ BCAD da dieta foi calculado em $\mathrm{mEq}(\mathrm{Na}+\mathrm{K})$ $(\mathrm{Cl}+\mathrm{S}) / \mathrm{kg}$ de $\mathrm{MS}$.

Os minerais $\mathrm{Ca}$ e $\mathrm{Mg}$ foram analisados por titulometria com Na2EDTA e o P por gravimetria, no Laboratório de Nutrição Animal do Departamento de Zootecnia da UFPR, segundo as normas da AOAC (1970).

Os minerais $\mathrm{Na}$ e $\mathrm{K}$ foram analisados por fotometria de chama, no Laboratório do Departamento de Solos da UFPR.

$\mathrm{O}$ mineral $\mathrm{Cl}$ foi analisado por titulometria com nitrato de prata e o $S$ por gravimetria segundo o método da AOAC (1970), no Laboratório do Curso de PósGraduação em Tecnologia de Alimentos da UFPR.

As amostras de sangue para as análises do CaT e do CaI foram colhidas em tubos de vidro a vácuo, usando heparina como anticoagulante, mediante punção da veia jugular e centrifugadas em uma centrífuga portátil, com 2800 rotações por minuto, durante 30 minutos, para a obtenção do plasma. As amostras de plasma foram congeladas em nitrogênio líquido até a análise. As colheitas foram realizadas no início da dieta, até doze horas após o parto, e um, dois, três e sete dias após o parto, denominados de período de colheitas $1,2,3,4,5$ e 6 , respectivamente.

A análise do $\mathrm{CaI}$ foi feita por eletrodo íon seletivo $\mathrm{Ca}++$ no aparelho ISELAB, no Laboratório do Hospital de Animais de Companhia da Pontifícia Universidade Católica do Paraná (PUCPR).

OCaT foi analisado por kit colorimétrico, utilizando o método da cresolftaleína-complexona, no Laboratório do Hospital de Animais de Companhia da PUCPR.

A urina foi colhida no período da manhã, durante a micção espontânea das vacas, em recipientes adequados e a leitura do $\mathrm{pH}$ foi realizada dentro de no máximo uma hora. A colheita foi realizada semanalmente, do início da dieta até o parto. Os dados foram agrupados em três períodos, sendo que o período 1 representou as amostras colhidas no início do experimento, o período 2 as amostras colhidas entre 20 e 11 dias antes do parto e o período 3 as amostras colhidas entre 10 e 1 dias antes do parto.

A análise do $\mathrm{pH}$ foi realizada por um medidor de pH portátil da marca Quimis, modelo Q400B.

Os dados de $\mathrm{pH}$ urinário, $\mathrm{CaT}$ e $\mathrm{CaI}$ foram analisados pelo programa computacional MSTAT (1989). Os dados de incidência de hipocalcemia subclínica foram analisados pelo programa computacional STATISTICA(1996), utilizando o teste exato de Fisher. A comparação das médias foi realizada pelo teste de Tukey a $5 \%$ de probabilidade.

Não houve diferença significativa entre os grupos para a produção de leite na lactação anterior e para o número de partos, por isso não foram utilizados como covariáveis para as análises das concentrações de $\mathrm{CaI}$ e CaT.

\section{Resultados e Discussão}

$\mathrm{O}$ CaT e o CaI não diferiram entre as dietas para nenhum dos períodos estudados e nem para a interação dieta e período de colheita, mas houve diferença significativa entre os períodos de colheita $(\mathrm{P}<0,01)$. $\mathrm{O}$ dia do parto (período 2) teve os menores valores tanto para o CaT quanto para o CaI (Tabela 2).

Resultados semelhantes foram observados por Gaynor et al. (1989) e Setti et al. (1998), para CaT, e por Campos et al. (1998) e Setti et al. (2001a), para CaT e CaI, que também não encontraram diferenças nos níveis de $\mathrm{Ca}$ entre os grupos aniônicos e catiônicos à parição. Setti et al. (1998) e Setti et al. (2001a), assim como neste trabalho, não observaram casos de paresia da parturiente nas vacas estudadas, enquanto Campos et al. (1998) observaram apenas um caso em uma vaca primípara. Baseado no trabalho de Block (1984), onde as vacas alimentadas com dieta catiônica, que não apresentaram paresia da parturiente, tiveram concentração de CaT normal durante três dias antes e três dias após o parto, pode-se afirmar que a não ocorrência de paresia da parturiente nestes trabalhos corrobora o fato de não se encontrar diferenças entre os grupos catiônicos e aniônicos.

Resultados diferentes deste estudo foram encon-

R. Bras. Zootec., v.32, n.5, p.1259-1265, 2003 
Tabela 2 - Médias e desvios-padrão da concentração plasmática de cálcio total (CaT) e de cálcio iônico (Cal) para os grupos 1, 2 e 3, com BCAD de +122,1, -8,82 e-110,41, respectivamente, nos períodos de colheita

Table 2 - Means and standard deviations of plasma concentrations of total calcium (tCa) and ionized calcium (iCa) for groups 1 , 2 and 3, with $D C A B+122.1,-8.82$ and -110.41 , respectively, in the sampling periods

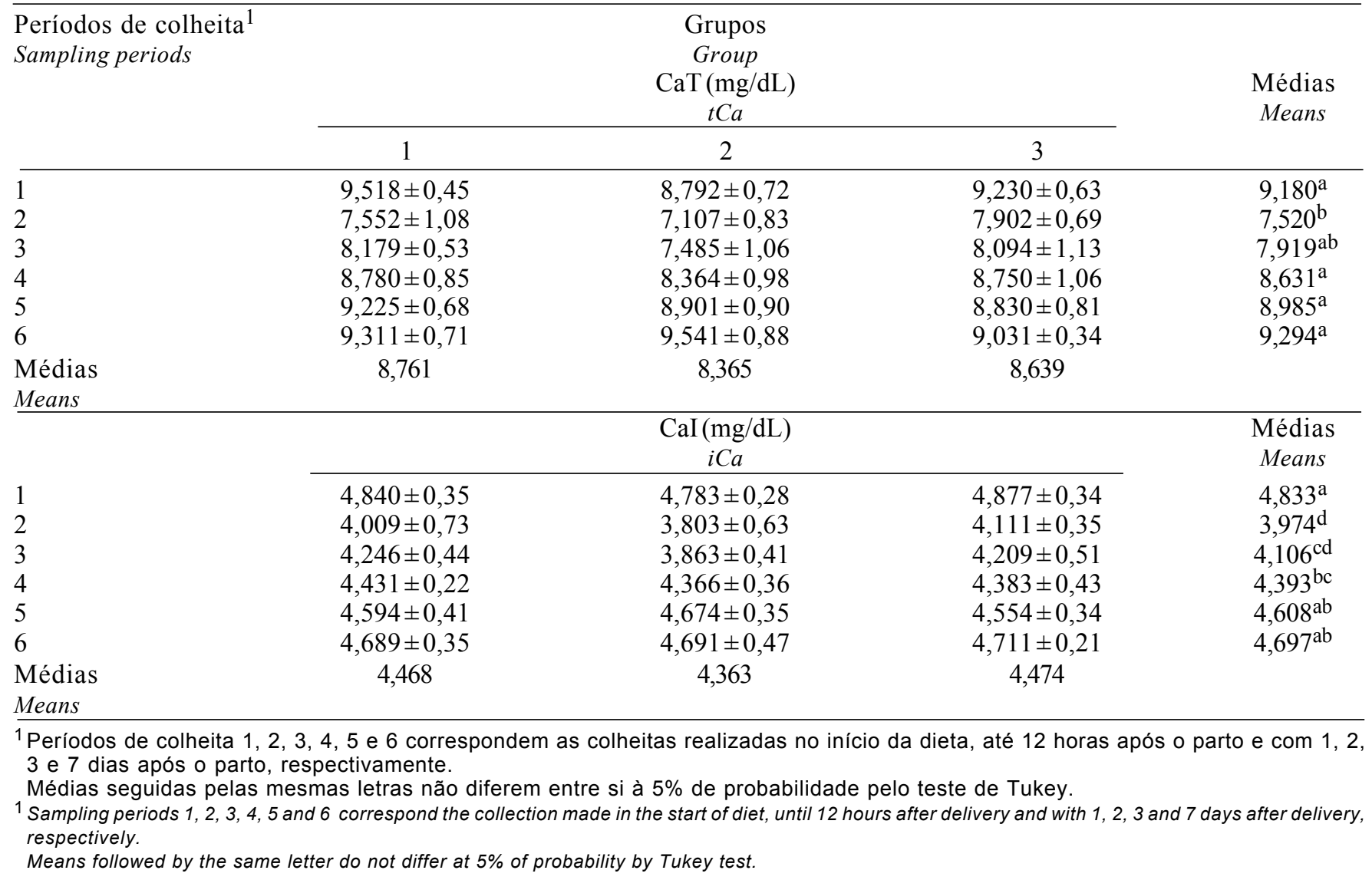

trados por diversos autores trabalhando com CaT (Block, 1984; Goff et al., 1991; Tucker et al., 1992; Goff \& Horst, 1997; Goff \& Horst, 1998), CaI (Moore et al., 2000) ou CaT e CaI (Oetzel et al., 1988; Joyce et al., 1997), que encontraram maiores concentrações de $\mathrm{Ca}$ ao parto com o uso da dieta aniônica. Estes trabalhos tiveram casos de paresia da parturiente e, então, conseguiram demonstrar melhor o efeito preventivo da dieta aniônica sobre a queda da concentração de $\mathrm{Ca}$ à parição.

$\mathrm{O} \mathrm{pH}$ urinário diferiu significativamente entre as dietas $(\mathrm{P}<0,05)$, períodos de colheita $(\mathrm{P}<0,01)$ e interação dieta e período de colheita $(\mathrm{P}<0,05)$. Os valores médios de $\mathrm{pH}$ urinário podem ser visualizados na Figura 1.

A diminuição do $\mathrm{pH}$ urinário demonstrou que a dieta aniônica foi capaz de induzir acidose metabólica suave nas vacas. Este efeito tem sido observado por diversos autores (Goff et al., 1991; Tucker et al., 1992; Joyce et al., 1997; Goff \& Horst, 1997; Campos et al., 1998; Goff \& Horst, 1998; Vagnoni \& Oetzel,

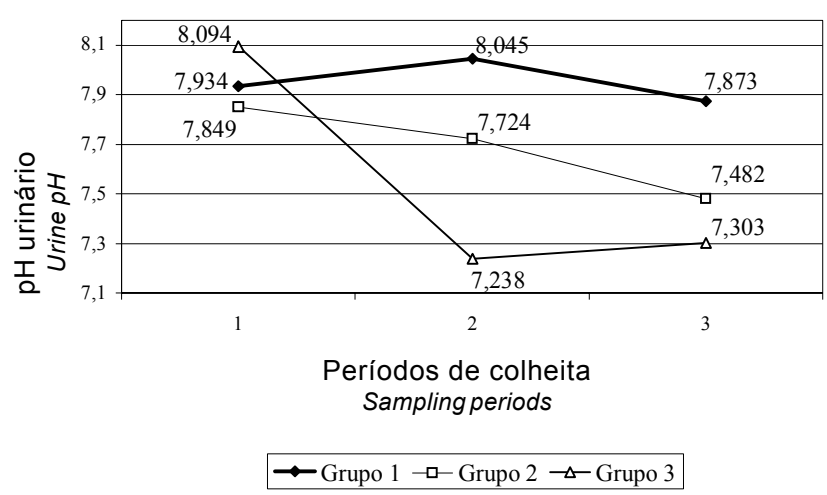

${ }^{1}$ Períodos de colheita 1,2 e 3 correspondem as colheitas
realizadas no início da dieta, entre 11 e 20 dias antes do parto
e entre 10 dias antes do parto e o parto.
${ }^{1}$ Sampling periods 1,2 and 3 correspond the collection made in the start
of diet, between 11 and 20 days before the delivery and between 10 days
before the delivery and the delivery, respectively.

Figura 1 - Valores de $\mathrm{pH}$ urinário dos grupos 1, 2 e 3, com BCAD de $+122,1,-8,82$ e $-110,41$, respectivamente, nos períodos de colheitas.

Figure 1 - Urine $p H$ of group1, 2 and 3, with $D C A B+122.1$, 8.82 and -110.41 , respectively, in the sampling period. 
1998; Moore et al., 2000; Setti et al., 2001b).

Não houve diferença significativa para a ocorrência de hipocalcemia subclínica entre os grupos ( $P>0,05$, teste exato de Fisher), mas houve tendência $(\mathrm{P}=0,1026)$ do grupo 3 ter maior incidência de hipocalcemia subclínica que o grupo 1 , quando medida pela concentração de CaI (Tabela 3). Este resultado é contrário ao esperado quando se utiliza a dieta aniônica.

Embora as médias de produção de leite na lactação anterior não tenham sido estatisticamente diferentes entre os três grupos, a distribuição das vacas de acordo com os níveis de produção (Figura 2) mostra que o grupo 1 teve o maior número de animais com menos de 7.000 $\mathrm{kg}$ de leite produzidos na lactação anterior. Estes são menos susceptíveis a paresia da parturiente e a hipocalcemia subclínica e contribuíram para a menor incidência de hipocalcemia subclínica no grupo 1.

Os valores de $\mathrm{pH}$ urinário deste trabalho foram acima daqueles recomendados por Jardon (1995), Davidson et al. (1995) e Moore et al. (2000), sugerindo que a acidose desenvolvida pelas vacas deste estudo não foi suficiente para prevenir a ocorrência de hipocalcemia subclínica. Moore et al. (2000) conseguiram prevenir a hipocalcemia subclínica com um $\mathrm{pH}$ urinário de 6,01 para o grupo aniônico durante o período pré-parto. Neste traba1ho, o pH urinário foi de 7,2 e 7,3 para 2 e 1 semanas antes do parto, respectivamente, para o grupo 3 . Porém, as médias de CaT e CaI das vacas deste grupo (mais aniônico) foram normais para todos os períodos estudados, enquanto que para o grupo 2 as médias foram abaixo do limite normal para $\mathrm{CaT}$ e CaI no parto e um dia após.

Oetzel et al. (1988) colocaram que o efeito preventivo da dieta aniônica sobre a hipocalcemia subclínica só ocorreu quando a dieta apresentou altos níveis de $\mathrm{Ca}(1,2 \%)$ na dieta. No presente estudo, o nível de Ca na dieta aniônica foi de $0,70 \%$.

Contudo, os valores mais baixos de $\mathrm{CaI}$ e de CaT foram, respectivamente, de 2,56 e $5,18 \mathrm{mg} / \mathrm{dL}$ para o grupo $1,3,06$ e $5,52 \mathrm{mg} / \mathrm{dL}$ para o grupo $2 \mathrm{e}$ 3,58 e $7,08 \mathrm{mg} / \mathrm{dL}$ para o grupo 3, indicando que a hipocalcemia subclínica foi mais severa no grupo catiônico. Este resultado está de acordo com os de Gaynor et al. (1989), que também não encontraram diferença na concentração de $\mathrm{CaT}$ entre os grupos, mas colocaram que todas as vacas do grupo mais aniônico mantiveram a concentração de CaT acima de $6,2 \mathrm{mg} / \mathrm{dL}$, o que não ocorreu com os demais grupos.

Muitos trabalhos têm mostrado a redução do $\mathrm{pH}$ urinário pelo fornecimento de sais aniônicos, mas o valor do BCAD e os valores de Ca obtidos no momento do parto variam muito e é difícil estabelecero valor ideal do BCAD e do pH urinário que mantém a concentração de Ca acima dos valores normais (Tabela 4).

A diversidade encontrada nos resultados de trabalhos com BCAD revela a complexidade envolvida no controle do equilíbrio ácido-básico em ruminantes e a influência deste na homeostasia do Ca. Isto acaba limitando as conclusões a respeito do papel do BCAD no metabolismo do Ca.

Diferenças na resposta individual das vacas podem explicar os resultados inesperados de alguns trabalhos, principalmente quando se utiliza um pequeno número de animais.

Enquanto o principal defeito metabólico que leva à paresia da parturiente não é descoberto, a utilização da dieta aniônica continua sendo o principal método para a sua prevenção.

Tabela 3 - Incidência de hipocalcemia subclínica nos grupos 1, 2 e 3, com BCAD de +122,1, -8,82 e $-110,41$, respectivamente, segundo os valores de cálcio total (CaT) e de cálcio iônico (Cal)

Table 3 - Incidence of subclinic hypocalcemia in the groups 1, 2 and 3, with $D C A B+122.1,-8.82$ and -110.41 , respectively, according to the concentrations of total calcium ( $t \mathrm{Ca}$ ) and ionized calcium (iCa)

\begin{tabular}{|c|c|c|}
\hline \multirow[t]{2}{*}{$\begin{array}{l}\text { Grupos } \\
\text { Group }\end{array}$} & \multicolumn{2}{|c|}{$\begin{array}{c}\text { Hipocalcemia subclínica }^{1} \\
\text { Subclinic hypocalcemia }\end{array}$} \\
\hline & $\begin{array}{l}\mathrm{CaT} \\
t C a\end{array}$ & $\begin{array}{l}\mathrm{CaI} \\
i \mathrm{Ca}\end{array}$ \\
\hline 1 & $2 / 7(29 \%)$ & $2 / 7(29 \%)$ \\
\hline 2 & $4 / 7(57 \%)$ & $4 / 7(57 \%)$ \\
\hline 3 & $4 / 7(57 \%)$ & $6 / 7(86 \%)$ \\
\hline
\end{tabular}

${ }^{1}$ Número de vacas com hipocalcemia subclínica / total de vacas do grupo.

${ }^{1}$ Number of cows with subclinic hypocalcemia / total cows in the group. 
Tabela 4 - Cálcio total (CaT), cálcio iônico (Cal) e pH urinário de diversos trabalhos com BCAD Table 4 - Total calcium ( $t C a)$, ionized calcium (iCa) and urinary $\mathrm{pH}$ of different researches with DCAB

\begin{tabular}{|c|c|c|c|c|c|c|c|c|}
\hline \multirow[t]{2}{*}{$\begin{array}{l}\text { Autores } \\
\text { Authors }\end{array}$} & \multicolumn{4}{|c|}{$\begin{array}{l}\text { Grupo aniônico } \\
\text { Anionic group }\end{array}$} & \multicolumn{4}{|c|}{$\begin{array}{l}\text { Grupo catiônico } \\
\text { Cationic group }\end{array}$} \\
\hline & $\begin{array}{r}\mathrm{BCAD}^{1} \\
D C A B\end{array}$ & $\begin{array}{r}\mathrm{CaT}^{2} \\
t C a\end{array}$ & $\begin{array}{r}\mathrm{CaI}^{2} \\
i C a\end{array}$ & $\begin{array}{r}\mathrm{pH}^{3} \\
\mathrm{pH}\end{array}$ & $\begin{array}{r}\text { BCAD } \\
D C A B\end{array}$ & $\begin{array}{r}\mathrm{CaT} \\
t C a\end{array}$ & $\begin{array}{l}\mathrm{CaI} \\
i \mathrm{Ca}\end{array}$ & $\begin{array}{l}\mathrm{pH} \\
\mathrm{pH}\end{array}$ \\
\hline Block(1984) & $-128,5$ & $>8,0$ & - & - & $+330,5$ & 6,2 & - & - \\
\hline Oetzel et al. (1988) & -75 & 8,4 & 4,05 & - & +180 & 7,4 & 3,56 & - \\
\hline Fredeen et al. $(1988)^{4}$ & +7 & - & - & 5,59 & +900 & - & - & 8,11 \\
\hline Gaynor et al. $(1989)^{4}$ & +220 & 7,01 & - & - & +1.258 & 6,43 & - & - \\
\hline Goff et al. (1991) & -228 & 7,63 & - & 7,2 & +978 & 6,58 & - & 8,3 \\
\hline Tucker et al. (1992) & -30 & 8,16 & - & 6,14 & +90 & 6,72 & - & 8,22 \\
\hline Wang \& Beede (1992) & -428 & - & - & 5,93 & +69 & - & - & 7,88 \\
\hline Abu Damir et al.(1994) & -35 & 8,8 & 4,4 & - & +779 & 7 & 3,44 & - \\
\hline Phillippo et al. (1994) & -8 & 9,36 & 4,8 & - & +534 & 8,04 & 4,64 & - \\
\hline Goff \& Horst (1997) & -54 & 6,9 & - & 5,75 & +461 & 5,22 & - & 8,17 \\
\hline Joyce et al. (1997) & -70 & - & 4,31 & $7,19^{5}$ & +350 & - & 3,87 & $8,4^{5}$ \\
\hline Goff \& Horst (1998) & $\mathrm{HCl}^{6}$ & 6,69 & - & - & $\mathrm{s} / \mathrm{HCl}$ & 5,33 & - & - \\
\hline Campos et al. (1998) & -75 & 7,81 & 4,33 & 5,92 & +186 & 7,89 & 4,35 & 7,76 \\
\hline Vagnoni \& Oetzel (1998) & -63 & - & - & 7,2 & +203 & - & - & 8,33 \\
\hline Setti et al. (1998) & +90 & 8,59 & - & - & +180 & 8,56 & - & - \\
\hline Moore et al. (2000) & -150 & - & 4,35 & 6,01 & +150 & - & 3,67 & 7,95 \\
\hline Setti et al. $(2001 \mathrm{a} / \mathrm{b})$ & $-50,8$ & 7,88 & 4,13 & $6^{7}$ & $+146,8$ & 8,13 & 4,36 & $7,9^{7}$ \\
\hline
\end{tabular}

${ }_{1} \mathrm{mEq}(\mathrm{Na}+\mathrm{K})-(\mathrm{Cl}+\mathrm{S}) / \mathrm{kg}$ de $\mathrm{MS}(\mathrm{mEq}(\mathrm{Na}+\mathrm{K})-(\mathrm{Cl}+\mathrm{S}) / \mathrm{kg}$ of $\mathrm{DM})$.

$2 \mathrm{mg} / \mathrm{dL}$ no dia do parto ( $\mathrm{mg} / \mathrm{dL}$ on the delivery day).

$3 \mathrm{pH}$ urinário da semana anterior ao parto ou conforme a legenda (Urinary $\mathrm{pH}$ of one week before the delivery or legend).

$4 \mathrm{mEq}(\mathrm{Na}+\mathrm{K}-\mathrm{Cl}) / \mathrm{kg}$ de $\mathrm{MS}$ ( $\mathrm{mEq}(\mathrm{Na}+\mathrm{K}-\mathrm{Cl}) / \mathrm{kg}$ of $\mathrm{DM})$.

5 Dois dias antes do parto (Two days before the delivery day).

$61,5 \mathrm{Eq}$ de $\mathrm{HCl}$ por dia (1.5 Eq of $\mathrm{HCl}$ per day).

72 semanas antes do parto (Two week before the delivery day).

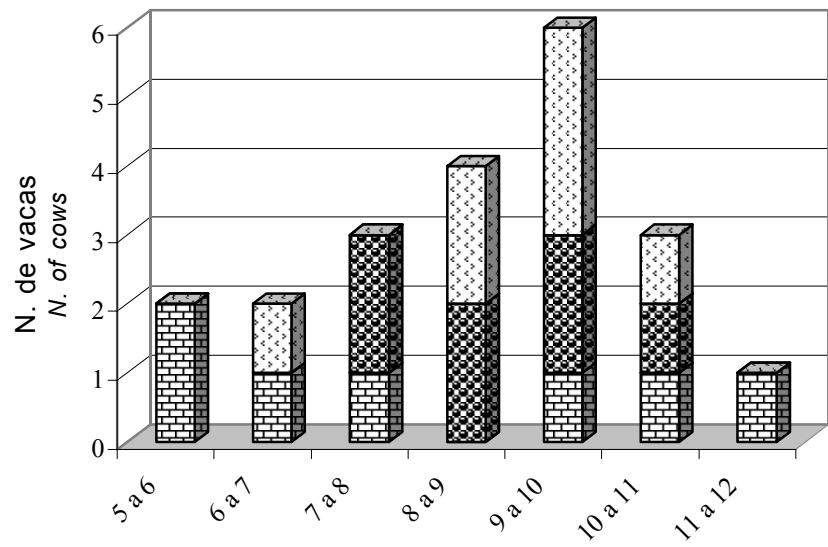

Nível de produção de leite $(x 100 \mathrm{~kg})$ Milk production level $(x 100 \mathrm{~kg})$

国 Grupo 1 图Grupo 2 G Grupo 3

Figura 2 - Distribuição das vacas dos grupos 1, 2 e 3, com BCAD de $+122,1,-8,82$ e $-110,41$, respectivamente, segundo o nível de produção.

Figure 2 - Cows allocations of groups 1,2 and 3, with DCAB $+122.1,-8.82$ and -110.41 , respectively, in production levels.

\section{Conclusões}

A redução no balanço catiônico-aniônico da dieta induziu acidose metabólica suave nas vacas, mas não afetou os níveis de cálcio total, cálcio iônico e a incidência de hipocalcemia subclínica entre os três grupos estudados.

\section{Agradecimento}

Ao CNPq, pela bolsa concedida.

À Nuvital Nutrientes Ltda, pela doação dos sais aniônicos.

Ao médico veterinário Marcos Epp, proprietário da Chácara Vitória.

\section{Literatura Citada}

ABU DAMIR, H.; PHILLIPPO, M.; THORP, B.H. et al. Effects of dietary acidity on calcium balance and mobilization, bone morphology and 1,25 dihydroxyvitamin $\mathrm{D}$ in prepartal dairy cows. Research Veterinary Science, v.56, p.310-318, 1994.

ASSOCIATION OF OFFICIAL ANALYTICAL CHEMISTS AOAC. Official methods of analysis. 11.ed. Washington, D.C.: $1970.1075 p$. 
BECK, N.; WEBSTER, S.K. Effects of acute metabolic acidosis on parathyroid hormone action and calcium mobilization. American Journal of Physiology, v.230, n.1, p.127-131, 1976.

BLOCK, E. Manipulating dietary anions and cations for prepartum dairy cows to reduce incidence of milk fever. Journal of Dairy Science, v.67, n.12, p.2939-2948, 1984.

BLOCK, E. Manipulation of dietary cation-anion difference on nutritionally related production diseases, productivity, and metabolic responses of dairy cows. In: SIMPÓSIO INTERNACIONAL DE PRODUÇÃO DE RUMINANTES, 1., 1994, Maringá. Anais... Maringá: Sociedade Brasileira de Zootecnia, 1994. p.21-48.

CAMPOS, J.M.; GRAÇA, D.S.; VALADARES FILHO, S.C. et al. Balanço dietético cátion-ânion na alimentação de vacas leiteiras, no pré-parto. II - Metabolismo mineral. In: REUNIÃO ANUAL DA SOCIEDADE BRASILEIRA DE ZOOTECNIA, 35., 1998, Botucatu. Anais... Botucatu: Sociedade Brasileira de Zootecnia, 1998. p.542-544.

CURTIS, C.R.; ERB, H.N.; SNIFFEN, C.J. et al. Association of parturient hypocalcemia with eight periparturient disorders in Holstein cows. Journal of the American Veterinary Medical Association, v.183, n.5, p.559-561, 1983.

DAVIDSON, J.; RODRIGUEZ, L.; PILBEAM, T. et al. Urine pH check helps avoid milk fever. Hoard's Dairyman, v.140, p.634, 1995.

FREDEEN, A.H.; DEPETERS, E.J.; BALDWIN, R.L. Characterization of acid base disturbances and effects on calcium and phosphorus balances of dietary fixed ions in pregnant or lactating does. Journal of Animal Science, v.66, p.159173, 1988.

GAYNOR, P.J.; MUELLER, F.J.; MILLER, J.K. et al. Parturient hypocalcemia in Jersey cows fed alfalfa haylage-based diets with different cation to anion rations. Journal of Dairy Science, v.72, n.10, p.2525-2531, 1989.

GOERING, H.K.; VAN SOEST, P.J. Forage fiber analysis: apparatus reagents, procedures and some applications. Washington, D.C.: Agriculture Handbook, 1970. 379p.

GOFF, J.P. Cation-anion difference of diets and its influence on milk fever and subsequent lactation: the good and the bad news. In: CORNELL NUTRITION CONFERENCE FOR FEED MANUFACTURERS, 54., 1992, Ithaca. Proceedings... Ithaca: Cornell University, 1992. p.148-167.

GOFF, J.P.; HORST, R.L. Effects of the addition of potassium or sodium, but not calcium, to prepartum rations on milk fever in dairy cows. Journal of Dairy Science, v.80, n.1, p.176-186, 1997.

GOFF, J.P.; HORST, R.L. Use of hydrochloric acid as a source of anions for prevention of milk fever. Journal of Dairy Science, v.81, n.11, p.2874-2880, 1998.

GOFF, J.P.; HORST, R.L.; MUELLER, F.J et al. Addition of chloride to a prepartal diet high in cations increases 1,25 dihydroxyvitamin $\mathrm{D}$ response to hypocalcemia preventing milk fever. Journal of Dairy Science, v.74, n.11, p.38633871, 1991.

JARDON, P.W. Using urine $\mathrm{pH}$ to monitor anionic salt programs. Compendium on Continuing Education for the Practicing Veterinarian, v.17, p.860-862, 1995.

JOYCE, P.W.; SANCHEZ, W.K.; GOFF, J.P. Effect of anionic salts in prepartum diets based on alfalfa. Journal of Dairy Science, v.80, n.11, p.2866-2875, 1997.

MOORE, S.J.; VANDEHAAR, M.J.; SHARMA, B.K. et al. Effects of altering dietary cation-anion difference on calcium and energy metabolism in peripartum cows. Journal of Dairy Science, v.83, n.9, p.2095-2104, 2000.
NATIONAL RESEARCH COUNCIL - NRC. Nutrient requirements of dairy cattle. 7.ed. Washington, D.C.: National Academy Press, 2001. 381p.

OETZEL, G.R.; OLSON, J.D.; CURTIS, C.R. et al. Ammonium chloride and ammonium sulfate for prevention of parturient paresis in dairy cows. Journal of Dairy Science, v.71, n. 12, p.3302-3309, 1988.

ORTOLANI, E.L. Aspectos clínicos, epidemiológicos e terapêuticos da hipocalcemia de vacas leiteiras. Arquivo Brasileiro de Medicina Veterinária e Zootecnia, v.47, n.6, p.799-808, 1995.

PHILLIPPO, M.; REID, G.W.; NEVISON, I.M. Parturient hypocalcemia in dairy cows: effects of dietary acidity on plasma minerals and calciotropic hormones. Research Veterinary Science, v.56, p.303-309, 1994.

SANCHEZ, W.K.; BLAUWIEKEL, R. Prevention of milk fever by application of the dietary cation-anion balance concept. Disponível em: <http://coopext.cahe.wsu.edu/ infopub/eb1783/eb1783.html> Acesso em: 17 nov. 1999.

SETTI, M.C.; SCHALCH, E.; ZANETTI, M.A. Estudo do balanço cátion-aniônico da dieta no desempenho de vacas Holandesas. Revista Brasileira de Zootecnia, v.27, n.6, p.1241-1247, 1998.

SETTI, M.C.; VIEIRA, P.F.; ZANETTI, M.A. et al. Balanço cátion-aniônico da dieta (BCAD) para vacas da raça holandesa, nas concentrações dos macrominerais no sangue. In: REUNIÃO ANUAL DA SOCIEDADE BRASILEIRA DE ZOOTECNIA, 38., 2001, Piracicaba. Anais... Piracicaba: Sociedade Brasileira de Zootecnia, 2001a. p.1311-1312.

SETTI, M.C.; VIEIRA, P.F.; ZANETTI, M.A. et al. Balanço cátion-aniônico da dieta (BCAD) para vacas da raça holandesa, no estado ácido-base no sangue e na urina. In: REUNIÃO ANUAL DA SOCIEDADE BRASILEIRA DE ZOOTECNIA, 38., 2001, Piracicaba. Anais... Piracicaba: Sociedade Brasileira de Zootecnia, 2001b. p.1307-1309.

STEWART, P.A. Modern quantitative acid-base chemistry. Canadian Journal of Physiology and Pharmacology, v.61, p.1444-1461, 1983.

TAKAGI, H.; BLOCK, E. Effects of various dietary cationanion balances on response to experimentally induced hypocalcemia in sheep. Journal of Dairy Science, v.74, n.12, p.4215-4224, 1991.

TUCKER, W.B.; HOGUE, J.F.; ADAMS, G.D. et al. Influence of dietary cation-anion balance during the dry period on the occurrence of parturient paresis in cows fed excess calcium. Journal of Animal Science, v.70, n.4, p.1238-1250, 1992.

VAGNONI, D.B.; OETZEL, G.R. Effects of dietary cationanion difference on the acid-base status of dry cows. Journal of Dairy Science, v.81, n.6, p.1643-1652, 1998.

WANG, C.; BEEDE, D.K. Effects of ammonium chloride and sulfate on acid-base status and calcium metabolism of dry Jersey cows. Journal of Dairy Science, v.75, n.3, p.820828, 1992.

WANG, C.; VÉLEZ, J.S.; RISCO, C.A. Avanços recentes na prevenção da paresia do parto nas vacas leiteiras. Compêndio de Educação Continuada, v.1, n.1, p.63-69, 1996.

R. Bras. Zootec., v.32, n.5, p.1259-1265, 2003

Recebido em: 03/09/02 Aceito em: 15/01/03 